\title{
Treatment of Saos-2 osteosarcoma cells with diallyl trisulfide is associated with an increase in calreticulin expression
}

\author{
WEN-PENG XIE $^{1 *}$, YUE ZHANG $^{1 *}$, YONG-KUI ZHANG $^{2}$, GANG LI $^{1,2}$, \\ JIAN XIN ${ }^{2}$, RONG-XIU BI ${ }^{1,2}$ and CHUAN-JIE LI $^{3}$
}

\begin{abstract}
${ }^{1}$ Department of Orthopedics, First Clinical Medical College, Shandong University of Traditional Chinese Medicine, Jinan, Shandong 250355; ${ }^{2}$ Department of Orthopedics, Affiliated Hospital of Shandong University of Traditional Chinese Medicine, Jinan, Shandong 250014; ${ }^{3}$ Department of Orthopedics, Laiwu Central Hospital of Xinwen Mining Group, Laiwu, Shandong 271103, P.R. China
\end{abstract}

Received October 19, 2017; Accepted February 22, 2018

DOI: $10.3892 /$ etm.2018.6037

\begin{abstract}
Diallyl trisulfide (DATS) is a natural organic sulfur compound that may be isolated from garlic and has strong anticancer activity. DATS has been demonstrated to upregulate the expression of calreticulin (CRT) in various types of human cancers, which is associated with the prognosis of cancer and its response to therapy. However, whether DATS has the same effect on human osteosarcoma cells is not known. Therefore, in the present study, Saos-2 human osteosarcoma cells were cultured with different concentrations of DATS $(0,25,50$ and $100 \mu \mathrm{mol} / \mathrm{l}$ ) for $24 \mathrm{~h}$, or with $50 \mu \mathrm{mol} / 1 \mathrm{DATS}$ for different time periods $(0,12,24$ and $36 \mathrm{~h})$. Reverse transcription-quantitative polymerase chain reaction (RT-qPCR), western blotting and immunofluorescent staining were used to detect CRT mRNA and protein in the Saos-2 cells. Exposure to DATS changed the morphology and inhibited the growth of the Saos-2 cells, and its effects appeared to be concentration- and exposure time-dependent. The optimum concentration and exposure time of DATS were $50 \mu \mathrm{mol} / 1$ and $24 \mathrm{~h}$, respectively. The levels of CRT mRNA and protein in the Saos-2 cells were significantly upregulated following exposure to DATS. The upregulation of CRT expression by DATS may be a mechanism underlying the ability of DATS to inhibit the growth of human osteosarcoma Saos-2 cells.
\end{abstract}

Correspondence to: Professor Yong-Kui Zhang, Department of Orthopedics, Affiliated Hospital of Shandong University of Traditional Chinese Medicine, 16369 Jingshi Road, Jinan, Shandong 250014, P.R. China

E-mail: xiaoniu0928@163.com

*Contributed equally

Abbreviations: CRT, calreticulin; DATS, diallyl trisulfide; RT-qPCR, reverse transcription-quantitative polymerase chain reaction

Key words: diallyl trisulfide, osteosarcoma, Saos-2, calreticulin, inhibitory effects

\section{Introduction}

Osteosarcoma is one of the most common malignancies in adolescents. The incidence rate of all people has been reported to be 6-8/1 million (1-3), accounting for $2.4 \%$ of all malignancies and $60 \%$ of all malignant bone tumors $(4,5)$. Furthermore, the incidence of osteosarcoma has been rising in recent years (6). Osteosarcoma originates from mesenchymal tissue and acquires the characteristics of strong invasiveness and early metastasis, which are associated with a poor prognosis $(3,4)$. Although neoadjuvant chemotherapy and surgical treatment are commonly used to treat osteosarcoma $(7,8)$, the curative effect and prognosis are relatively poor; the 5-year survival rate for patients with osteosarcoma without metastases is $<70 \%$ (9). Some patients are insensitive to chemotherapy, and others are not able to tolerate the side effects of chemotherapy, including myelosuppression, kidney toxicity and gastrointestinal reactions. Therefore, it is necessary to explore the pathogenesis of osteosarcoma further and to develop novel effective drugs with reduced side effects for these patients.

Calreticulin (CRT) is a multifunctional $\mathrm{Ca}^{2+}$-binding protein and an effector of the unfolded protein response, which participates in the regulation of intracellular $\mathrm{Ca}^{2+}$ homeostasis, protein folding and processing, antigen presentation, cellular differentiation and apoptosis (10). It is a molecular chaperone located in the endoplasmic reticulum (11). Previous studies have demonstrated that CRT expression is closely associated with the occurrence, development, diagnosis, prognosis and response to therapy of various tumors (11-19); CRT has been shown to promote the growth of certain tumors, but to inhibit the growth of others. A previous study conducted by the present research team (20) identified that there was a strong association between low expression levels of CRT and osteosarcoma growth and metastasis, which suggests that CRT may serve as a potential drug target for osteosarcoma.

Diallyl trisulfide (DATS) (21), a volatile oil-like bioactive compound derived from allium vegetables, has a variety of biological effects, including lipid-lowering, antibacterial, anti-inflammatory and immune-enhancing effects (22). DATS has previously exhibited strong anticancer effects, and is widely used as an anticancer and chemopreventive agent for 
certain tumors $(23,24)$. However, the effect of DATS on osteosarcoma and the underlying mechanism are largely unknown. A previous proteomic study conducted by the present research team (25) demonstrated that DATS suppressed Saos-2 human osteosarcoma cell proliferation by blocking cell cycle progression and inducing apoptosis in a dose- and time-dependent manner. In addition, nine upregulated proteins were detected as DATS-sensitive proteins, one of which was CRT. In the present study, the effects of DATS on Saos-2 cells were observed, and the level of CRT expression in the Saos-2 cells was investigated.

\section{Materials and methods}

Materials. DATS (98\% pure) was purchased from LKT Laboratories, Inc. (Minneapolis, MN, USA). Dulbecco's modified Eagle's medium (DMEM) and fetal bovine serum (FBS) were obtained from Gibco (Thermo Fisher Scientific, Inc., Waltham, MA, USA). Rabbit anti-human polyclonal CRT antibody (cat. no. ab227444) and mouse anti-human polyclonal $\beta$-actin antibody (cat. no. ab8227) were purchased from Abcam (Cambridge, UK). Fluorescein isothiocyanate (FITC)-labeled goat anti-rabbit immunoglobulin G antibody (FITC-IgG; heavy and light chain; cat. no. E031220-01) was obtained from EarthOx Life Sciences (Millbrae, CA, USA). Horseradish peroxidase (HRP)-conjugated goat anti-rabbit IgG antibodies (cat. no. sc-2004) were obtained from Santa Cruz Biotechnology, Inc. (Santa Cruz, CA, USA). The primer sequences were as follows: CRT upstream, 5'-TTGGAAGAG ATTGGGACT-3' and downstream, 5'-GCCAAAGTTATC ATAGGCATAGA-3'; $\beta$-actin upstream, 5'-CTCCCTGGA GAAGAGCTACGA-3' and downstream, 5'-CGATCCACA CGGAGTACTTGC-3' (Takara Biotechnology Co., Ltd., Dalian, China).

Cell culture. Saos-2 cells were obtained from the Institute of Biochemistry and Cell Biology at the Chinese Academy of Sciences (Shanghai, China). The cells were cultured in DMEM supplemented with $10 \% \mathrm{FBS}$ at $37^{\circ} \mathrm{C}$ in humidified air containing $5 \% \mathrm{CO}_{2}$. Cells were used in the logarithmic phase of growth throughout the study.

Immunofluorescent staining assay. The Saos- 2 cells $\left(\sim 5 \times 10^{5}\right)$ were treated with different concentrations of DATS $(0,25,50$ and $100 \mu \mathrm{mol} / \mathrm{l}$ ) for $24 \mathrm{~h}$, and with $50 \mu \mathrm{mol} / 1$ DATS for different time periods $(0,12,24$ and $36 \mathrm{~h})$ in 24 -well culture plates. Following treatment, the Sao- 2 cells were washed three times in PBS and then fixed in PBS containing 4\% formaldehyde for $30 \mathrm{~min}$ at room temperature. The cells were then washed twice in PBS and incubated for 30 min in PBS containing $0.1 \%$ Triton X-100. Next, the cells were blocked with $200 \mu 1$ 10\% normal goat serum (Gibco; Thermo Fisher Scientific, Inc.) diluted with PBS per well and incubated for $30 \mathrm{~min}$ at room temperature. Thereafter, the cells were incubated with $200 \mu 1$ primary CRT antibody (dilution, 1:75) per well in a moist chamber at $4^{\circ} \mathrm{C}$ overnight. After washing three times with PBS, the cells were incubated with goat anti-rabbit FITC-IgG antibody (dilution, 1:200) at room temperature for $30 \mathrm{~min}$. Following this, the cells were blocked with glycerol followed by incubation with DAPI for $5 \mathrm{~min}$ at room temperature.
The cells were then analyzed using a Leica DM4000B microscope (Leica Microsystems GmbH, Wetzlar, Germany). Images were captured and expression levels determined using the Image-Pro Plus image analysis system 7.0 (Media Cybernetics, Inc., Rockville, MD, USA). Cells in which the cytoplasm was stained green were considered to be CRT-positive cells.

Reverse transcription-quantitative polymerase chain reaction ( $R T-q P C R)$ assay. Following treatment with DATS as described for the immunofluorescent staining assay, total RNA was extracted from the Saos-2 cells using TRIzol reagent (Invitrogen; Thermo Fisher Scientific, Inc.) and synthesized into cDNA using the PrimeScript RT reagent kit (Takara Biotechnology Co., Ltd.) at $37^{\circ} \mathrm{C}$ for $15 \mathrm{~min}$ and $85^{\circ} \mathrm{C}$ for $5 \mathrm{sec}$. qPCR analysis was then performed using SYBR Premix Ex Taq II (Takara Biotechnology Co., Ltd.) with a LightCycler 480 system (Roche Diagnostics, Basel, Switzerland). A $25-\mu 1$ PCR reaction mixture contained $2 \mu \mathrm{l}$ cDNA, $12.5 \mu 1$ SYBR Premix Ex Taq II, $1 \mu 1$ each primer and $8.5 \mu 1$ diethyl pyrocarbonate water. Following an initial denaturation step at $95^{\circ} \mathrm{C}$ for $2 \mathrm{~min}, \mathrm{CRT}$ and $\mathrm{B}$-actin were amplified with 45 cycles at $95^{\circ} \mathrm{C}$ for $20 \mathrm{sec}, 60^{\circ} \mathrm{C}$ for $30 \mathrm{sec}$ and $68^{\circ} \mathrm{C}$ for $30 \mathrm{sec}$. The levels of $\beta$-actin were used as an internal control. All reactions were run in triplicate. The data output from the RT-qPCR experiments were then analyzed using the $2^{-\Delta \Delta C q}$ method (26).

Western blot analysis. Following treatment with DATS as described for the immunofluorescent staining assay, protein was extracted from the Saos-2 cells using radioimmunoprecipitation assay buffer (Beyotime Institute of Biotechnology, Haimen, China) containing phenylmethane sulfonyl fluoride. A BCA protein assay kit (Beyotime Institute of Biotechnology) was used to evaluate the total protein concentration. Protein samples $(30 \mu \mathrm{g} / \mathrm{lane})$ were separated by SDS-PAGE $(8 \%)$ and transferred to polyvinylidene difluoride membranes. The membranes were incubated overnight at $4^{\circ} \mathrm{C}$ with primary anti-CRT antibody (dilution, 1:1,000) followed by the HRP-conjugated goat anti-rabbit IgG secondary antibody (dilution, 1:20,000) at room temperature for $1 \mathrm{~h}$. The blots were then visualized using a chemiluminescent detection kit (Amersham; GE Healthcare Life Sciences, Chalfont, UK). A Typhoon PhosphorImager with ImageQuant TL software version 7.0 (both GE Healthcare Life Sciences) was used to quantify the protein. The antibody against $\beta$-actin served as an internal reference. The expression level of CRT protein in each sample was determined as follows: Expression level of CRT protein $=$ densitometric value of $\mathrm{CRT}$ protein/densitometric value of $\beta$-actin.

Statistical analysis. All data are expressed as the mean \pm standard error of the mean of three independent experiments. The statistical significance of differences in the expression of CRT mRNA and protein among multiple groups was determined using one-way analysis of variance followed by Scheffe's post hoc test. SPSS 19.0 software (IBM Corp., Armonk, NY, USA) was used for statistical analysis. $\mathrm{P}<0.05$ was considered to indicate a statistically significant difference. 


\section{Results}

DATS inhibits the growth of Saos-2 human osteosarcoma cells. Changes in the morphology and growth status of the Saos-2 cells were observed under an inverted microscope following culture with different concentrations of DATS $(0,25,50$ and $100 \mu \mathrm{mol} / \mathrm{l}$ ) for $24 \mathrm{~h}$, and with $50 \mu \mathrm{mol} / 1$ DATS for different exposure times $(0,12,24$ and $36 \mathrm{~h})$. In the DATS-treated cells, morphological deformations, including the shrinkage of cell bodies, loosening of intercellular gaps, cell lysis and condensation of nuclei were observed. In addition, a number of cells became round, floated and necrotic. Furthermore, these morphological changes appeared to be concentrationand exposure time-dependent phenomena. However, the cell morphology of the control group appeared normal (data not shown). Images of the Saos- 2 cells following treatment with DATS under these conditions have been presented by the present authors in a previous study (27).

DATS induces the expression of CRT mRNA in Saos-2 cells. The presence of CRT mRNA was detected in the Saos-2 cells treated with different concentrations of DATS for $24 \mathrm{~h}$ (Fig. 1A) and $50 \mu \mathrm{mol} / 1$ DATS for different exposure times (Fig. 1B). The results demonstrated that the expression level of CRT mRNA was significantly upregulated following culture with DATS, and the upregulation appeared to be concentration- and exposure time-dependent (Fig. 1).

DATS increases CRT protein levels in Saos-2 cells. To characterize the functional relevance of increased CRT expression in osteosarcoma, analysis of CRT protein levels in the Saos-2 cells following culture with different concentrations of DATS for $24 \mathrm{~h}$ and with $50 \mu \mathrm{mol} / \mathrm{l}$ DATS for different exposure times was conducted. In the immunofluorescent staining assay, CRT exhibited prominent nuclear localization and increased expression intensity following treatment with DATS (Fig. 2). The expression intensity of CRT appeared to increase as the concentration (Fig. 2A) and exposure time (Fig. 2B) increased.

Western blotting was conducted to support further evaluate the CRT protein content of the cells following culture with different concentrations of DATS for $24 \mathrm{~h}$ (Fig. 3A) and with $50 \mu \mathrm{mol} / 1$ DATS for different exposure times (Fig. 3B). Treatment with DATS significantly reduced the CRT protein levels. The levels of CRT protein detected by western blotting were consistent those indicated by immunofluorescent staining.

\section{Discussion}

Developments in neoadjuvant chemotherapy, surgical treatment and comprehensive treatments for osteosarcoma have provided significant benefits to the treatment of osteosarcoma in recent years. Neoadjuvant chemotherapy combined with surgery is currently the preferred treatment for osteosarcoma (28), following more than 40 years of development and improvement (29). This type of treatment has great advantages, including the ability to limit or shrink the primary lesion, increase the likelihood of surgical success, reduce the difficulty of surgery and increase the 5-year survival rate of patients with osteosarcoma $(28,29)$. However, it has numerous
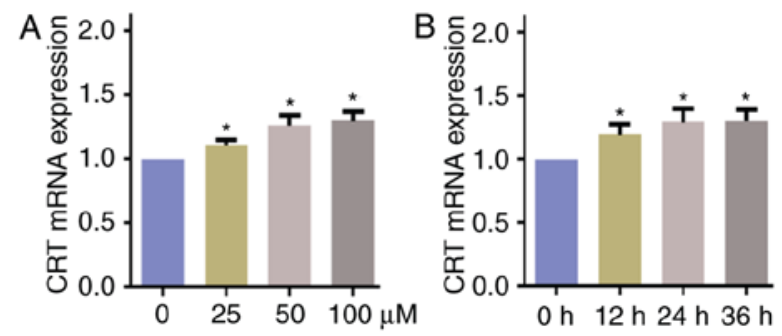

Figure 1. Detection of CRT mRNA in Saos-2 cells following treatment with DATS detected using reverse transcription-quantitative polymerase chain reaction with $\beta$-actin as a reference gene. CRT mRNA levels in Saos- 2 cells cultured with (A) different concentrations of DATS for $24 \mathrm{~h}$ and (B) $50 \mu \mathrm{M}$ DATS for different exposure times. Error bars represent the standard error of the mean. ${ }^{*} \mathrm{P}<0.05$ vs. control group. CRT, calreticulin; DATS, diallyl trisulfide.

A

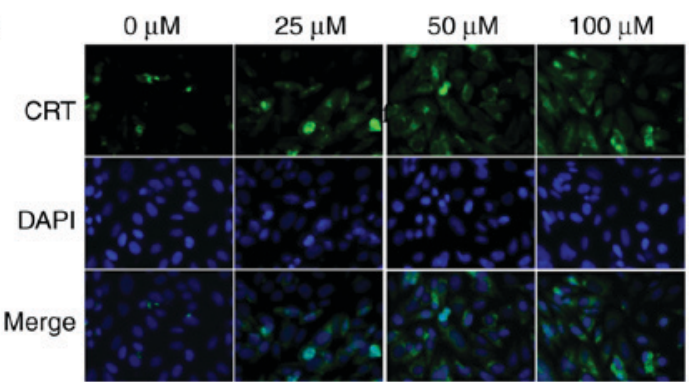

B

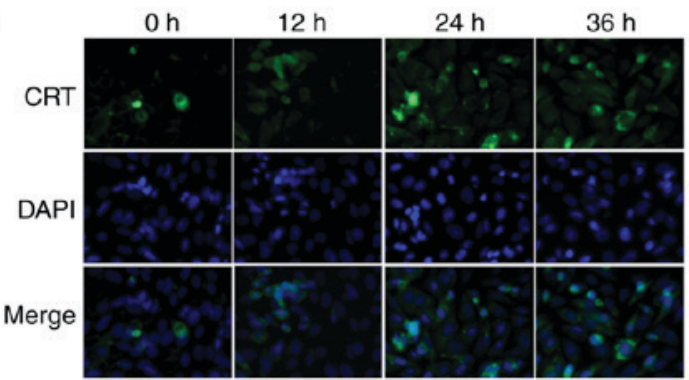

Figure 2. Effect of DATS on the expression of the cell surface differentiation marker CRT. CRT is indicated by immunofluorescence (green, upper panels). The DNA-intercalating dye DAPI was used to distinguish cell nuclei (blue, center panels). The lower panels display merged images to highlight the nuclear localization of CRT. Images for Saos-2 cells cultured with (A) different concentrations of DATS for $24 \mathrm{~h}$ and (B) $50 \mu \mathrm{M}$ DATS for different exposure times (magnification, x400). CRT, calreticulin.

side effects and complications, including myelosuppression, hepatorenal toxicity, serious gastrointestinal reactions and drug resistance (30). The development of neoadjuvant chemotherapy has slowed. Therefore, the exploration of new effective biomarkers, sensitive chemotherapeutic agents with reduced side effects and molecular therapeutic targets is necessary.

A large amount of research has been conducted on plant extracts, and many satisfactory results have been achieved. Garlic extract has attracted considerable attention due to its advantages of readily absorbed, non-polluting to the environment and widely available compared with traditional chemotherapeutic medicines. However, although the term garlic extract usually refers to a complex mixture of heterogeneous allyl organic sulfides, the present study investigated a specific component of garlic extract, namely DATS. DATS has been demonstrated to have good anticancer effects, with the ability to inhibit the 
A
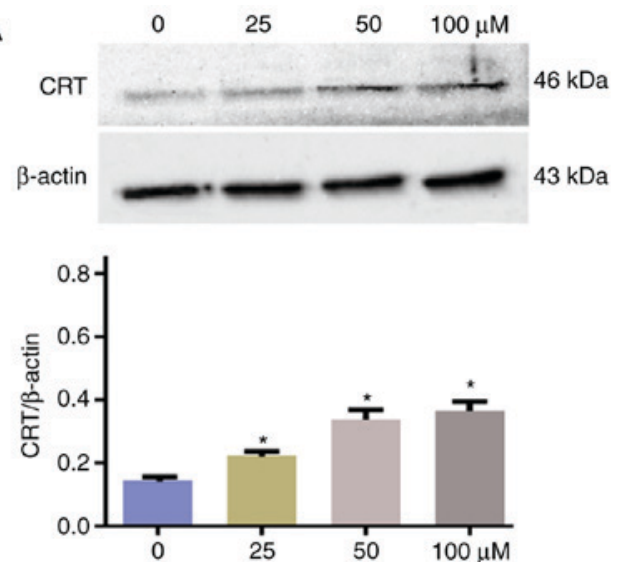

B
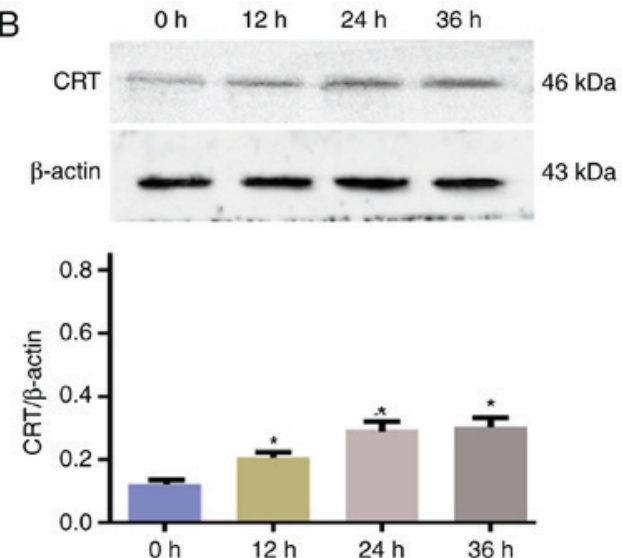

Figure 3. Western blot analysis of CRT expression level in Saos-2 cells following treatment with DATS. CRT protein levels in Saos-2 cells cultured with (A) different concentrations of DATS for $24 \mathrm{~h}$ and (B) $50 \mu \mathrm{M}$ DATS for different exposure times. $\beta$-actin was used as a loading control. Error bars represent the standard error of the mean. ${ }^{*} \mathrm{P}<0.05$ vs. control group.

proliferation and metastasis of various types of cancer, including prostate cancer (31), gastric cancer (32), lung cancer (33), breast cancer (34) and colon cancer (35). Oommen et al (36) reported that DATS promoted the apoptosis of SiHa cells by increasing the activity of caspase- $-3,-8$ and -9 , and downregulating the expression of poly (ADP-ribose) polymerase. Xu et al (37) revealed that C-Jun N-terminal kinase (JNK) activation and mitochondrial Bax translocation were involved in the apoptosis of SKOV3 cells induced by another component of garlic extract, allicin. Li et al (38) demonstrated that allicin inhibited the proliferation of Caco-2 cells by downregulating the expression of nuclear factor- $\mathrm{\kappa B}$ p65 and blocking the p38 and JNK signaling pathway. However, few studies investigating the effect of DATS on osteosarcoma and the underlying mechanism have been conducted.

A previous study conducted by the present research team demonstrated that DATS inhibited the proliferation of osteosarcoma cells and promoted their apoptosis in a dose- and time-dependent manner in vitro (25). Another study by the present research team (20) observed that the expression of CRT in patients with osteosarcoma was higher in the normal tissues surrounding the tumors than in the tumor tissues. In addition, CRT expression was greater in patients without metastasis than in those with metastasis, and in patients following chemotherapy than in those prior to chemotherapy. These results suggest that the CRT expression level is negatively associated with osteosarcoma malignancy. Therefore, further experiments to elucidate the mechanism of DATS on growth of human osteosarcoma were conducted in the present study.

The observation of Saos-2 cells under an inverted microscope following treatment with DATS revealed that DATS damaged the normal structure of the cells and inhibited their growth. These morphological changes appeared to be positively associated with the DATS concentration and exposure time, and indicate that DATS is able to inhibit the growth of osteosarcoma, consistent with previous study (27). Immunofluorescence staining, RT-qPCR and western blot analyses were conducted in the present study to detect CRT expression following the exposure of Saos-2 cells to DATS. The experimental results consistently demonstrated that DATS upregulated the expression of CRT in these cells, in an apparently concentration- and exposure time-dependent manner. Therefore, it is possible that the inhibition of osteosarcoma cell growth by DATS may occur via the upregulation of CRT expression. Furthermore, upregulating the expression of CRT via DATS treatment may be a possible therapeutic strategy for the effective management of osteosarcoma.

Although a number of studies on the association between CRT and malignant tumors have been conducted, the findings concerning the level and significance of CRT expression in cancer are not consistent in different types of tumors. Previous studies have demonstrated that CRT is upregulated in oral squamous cell carcinoma (11), pancreatic cancer (14), breast cancer (15), hepatocellular carcinoma (17), adrenocortical carcinomas (18) and gastric cancer (19). However, the results of a previous study by the present authors indicated that CRT was downregulated in osteosarcoma cells (20). In the present study, immunofluorescent staining, RT-qPCR and western blotting were conducted to detect the expression of CRT in Saos-2 osteosarcoma cells following culture with DATS at different concentrations and exposure times.

CRT has been demonstrated to serve an important role in inhibiting neovascularization and decreasing the blood supply to tumor tissue. In addition, CRT recruits macrophages to tumor cells to engulf and destroy the tumor cells, induces the production of killer lymphocytes and improves the ability of the immune system to kill tumor cells (39). Furthermore, CRT binds to the cluster of differentiation 47 receptor on the surface of certain innate immune cells, such as macrophages and dendritic cells, and increases the immune response to tumor cells (40). These previous observations and the results of the present study suggest that CRT may serve as a potential target for the development of therapeutics and preventive strategies for osteosarcoma.

The present study demonstrated that the treatment of Saos-2 osteosarcoma cells with DTS was associated with an increase in CRT expression. The possible mechanisms for this are as follows. Firstly, DATS may upregulate CRT expression by antagonizing the peroxisome proliferator-activated receptors that control the metabolic processes in various cells $(37,41)$. Secondly, DATS acts as a calcium 
antagonist and is able to influence the calcium channels on cell membranes (42). Therefore, it may upregulate CRT expression by regulating the calcium concentration in osteosarcoma cells.

However, the present study is a preliminary study in which only in vitro experiments were conducted. Further in-depth experiments using relevant animal models are required to clarify the curative effect and mechanism of DATS. In addition, the biological roles of CRT require further investigation in DATS-treated osteosarcoma cells in which CRT expression has been knocked down or overexpressed. In addition, the results require confirmation using other osteosarcoma cell lines, for example, HOS, U2OS and MG63 cells. Therefore, the investigation of the CRT-dependent molecular targets and signaling pathways will be the focus of future studies by the present research team.

In conclusion, the present study revealed a novel mechanism and potential molecular target of DATS in osteosarcoma cells. The results indicate that the known inhibitory effect of DATS on the growth of Saos-2 osteosarcoma cells may be mediated via upregulation of the expression of CRT. The present study provides a basis for the clinical use of DATS and suggests a novel molecular target for the treatment of osteosarcoma.

\section{Acknowledgements}

The authors thank Professor Yanli Ban, Qilu Hospital of Shandong University (Jinan, China), for linguistic advice.

\section{Funding}

The present study was funded by the National Science Foundation of Shandong Province (grant no. ZR2014HQ034) and the Medical Science Development Project of Shandong Province (grant no. 2014WS0432).

\section{Availability of data and materials}

The datasets used and/or analyzed during the current study are available from the corresponding author on reasonable request.

\section{Authors' contributions}

WPX and YZ performed most of the experiments and were major contributors in writing the manuscript. YKZ guided the design and implementation of the experiment. GL and JX analyzed and interpreted the experimental data. RXB and CJL conducted the immuofluorescence staining and processed the figures in the manuscript. All authors read and approved the final manuscript.

\section{Ethics approval and consent to participate}

Not applicable.

\section{Consent for publication}

Not applicable.

\section{Competing interests}

The authors declare that they have no conflicts of interest.

\section{References}

1. Chen N, Zhang R, Konishi T and Wang J: Upregulation of NRF2 through autophagy/ERK 1/2 ameliorates ionizing radiation induced cell death of human osteosarcoma U-2 OS. Mutat Res 813: 10-17, 2017.

2. Bielack SS, Hecker-Nolting S, Blattmann C and Kager L: Advances in the management of osteosarcoma. F1000 Res 5: 2767, 2016.

3. Friebele JC, Peck J, Pan X, Abdel-Rasoul M and Mayerson JL: Osteosarcoma: A meta-analysis and review of the literature. Am J Orthop (Belle Mead NJ) 44: 547-553, 2015.

4. Liu K, Sun X, Zhang Y, Liu L and Yuan Q: MiR-598: A tumor suppressor with biomarker significance in osteosarcoma. Life Sci 188: 141-148, 2017.

5. Taran SJ, Taran R and Malipatil NB: Pediatric osteosarcoma: An updated review. Indian J Med Paediatr Oncol 38: 33-43, 2017.

6. Chen L, Pei H, Lu SJ, Liu ZJ, Yan L, Zhao XM, Hu B and Lu HG: SPOP suppresses osteosarcoma invasion via PI3K/AKT/NF- $\mathrm{B}$ signaling pathway. Eur Rev Med Pharmacol Sci 22: 609-615, 2018.

7. He X, Gao Z, Xu H, Zhang Z and Fu P: A meta-analysis of randomized control trials of surgical methods with osteosarcoma outcomes. J Orthop Surg Res 12: 5, 2017.

8. Isakoff MS, Bielack SS, Meltzer P and Gorlick R: Osteosarcoma: Current treatment and a collaborative pathway to success. J Clin Oncol 33: 3029-3035, 2015.

9. Anderson ME: Update on survival in osteosarcoma. Orthop Clin North Am 47: 283-292, 2016.

10. Sun J, Mu H, Dai K and Yi L: Calreticulin: A potential anti-cancer therapeutic target. Pharmazie 72: 503-510,2017.

11. Harada K, Takenawa T, Ferdous T, Kuramitsu Y and Ueyama Y: Calreticulin is a novel independent prognostic factor for oral squamous cell carcinoma. Oncol Lett 13: 4857-4862, 2017.

12. Fucikova J, Truxova I, Hensler M, Becht E, Kasikova L, Moserova I, Vosahlikova S, Klouckova J, Church SE, Cremer I, et al: Calreticulin exposure by malignant blasts correlates with robust anticancer immunity and improved clinical outcome in AML patients. Blood 128: 3113-3124, 2016.

13. Obakan-Yerlikaya P, Arisan ED, Coker-Gurkan A, Adacan K, Ozbey U, Somuncu B, Baran D and Palavan-Unsal N: Calreticulin is a fine tuning molecule in epibrassinolide-induced apoptosis through activating endoplasmic reticulum stress in colon cancer cells. Mol Carcinog 56: 1603-1619, 2017.

14. Matsukuma S, Yoshimura K, Ueno T, Oga A, Inoue M, Watanabe Y, Kuramasu A,Fuse M, Tsunedomi R, Nagaoka S, et al: Calreticulin is highly expressed in pancreatic cancer stem-like cells. Cancer Sci 107: 1599-1609, 2016.

15. Zamanian M, Qader Hamadneh LA, Veerakumarasivam A, Abdul Rahman S, Shohaimi S and Rosli R: Calreticulin mediates an invasive breast cancer phenotype through the transcriptional dysregulation of p53 and MAPK pathways. Cancer Cell Int 16: $56,2016$.

16. Fucikova J, Becht E, Iribarren K, Goc J, Remark R, Damotte D, Alifano M, Devi P, Biton J, Germain C, et al: Calreticulin expression in human non-small cell lung cancers correlates with increased accumulation of antitumor immune cells and favorable prognosis. Cancer Res 76: 1746-1756, 2016.

17. Feng R, Ye J, Zhou C, Qi L, Fu Z, Yan B, Liang Z, Li R and Zhai W: Calreticulin down-regulation inhibits the cell growth, invasion and cell cycle progression of human hepatocellular carcinoma cells. Diagn Pathol 10: 149, 2015.

18. Yang MS, Wang HS, Wang BS, Li WH, Pang ZF, Zou BK, Zhang X, Shi XT, Mu DB, Zhang DX, et al: A comparative proteomic study identified calreticulin and prohibitin up-regulated in adrenocortical carcinomas. Diagn Pathol 8: 58, 2013.

19. Chen CN, Chang CC, Su TE, Hsu WM, Jeng YM, Ho MC, Hsieh FJ, Lee PH, Kuo ML, Lee H and Chang KJ: Identification of calreticulin as a prognosis marker and angiogenic regulator in human gastric cancer. Ann Surg Oncol 16: 524-533, 2009.

20. Zhang XH, Zhang Y, Xie WP, Sun DS, Zhang YK, Hao YK and Tan GQ: Expression and significance of calreticulin in human osteosarcoma. Cancer Biomark 18: 405-411, 2017.

21. Mikaili P, Maadirad S, Moloudizargari M, Aghajanshakeri S and Sarahroodi S: Therapeutic uses and pharmacological properties of garlic, shallot, and their biologically active compounds. Iran J Basic Med Sci 16: 1031-1048, 2013. 
22. Horn N, Miller G, Ajuwon KM and Adeola O: Garlic diallyl disulfide and diallyl trisulfide mitigates effects of pro-oxidant induced cellular stress and has immune modulatory function in LPS-stimulated porcine epithelial cells. J Anim Sci 95: 4045-4051, 2017.

23. Antony ML and Singh SV: Molecular mechanisms and targets of cancer chemoprevention by garlic-derived bioactive compound diallyl trisulfide. Indian J Exp Biol 49: 805-816, 2011.

24. Seki T, Hosono T, Hosono-Fukao T, Inada K, Tanaka R, Ogihara J and Ariga T: Anticancer effects of diallyl trisulfide derived from garlic. Asia Pac J Clin Nutr 17 (Suppl 1): S249-S252, 2008.

25. Zhang YK, Zhang XH, Li JM, Sun DS, Yang Q and Diao DM: A proteomic study on a human osteosarcoma cell line Saos-2 treated with diallyl trisulfide. Anticancer Drugs 20: 702-712, 2009.

26. Livak KJ and Schmittgen TD: Analysis of relative gene expression data using real-time quantitative PCR and the 2(-Delta Delta C(T)) method. Methods 25: 402-408, 2001

27. Zhang YK, Li JM, Wang DL and Chen YQ: Effects of diallyl trisulfide on cell cycle and apoptosis of human osteosarcoma cell line Saos-2. Tumor 33: 214-222, 2013 (In Chinese).

28. Yuan G, Chen J, Wu D and Gao C: Neoadjuvant chemotherapy combined with limb salvage surgery in patients with limb osteosarcoma of Enneking stage II: A retrospective study. Onco Targets Ther 10: 2745-2750, 2017.

29. Rosen G, Marcove RC, Caparros B, Nirenberg A, Kosloff C and Huvos AG: Primary osteogenic sarcoma: The rationale for preoperative chemotherapy and delayed surgery. Cancer 43: 2163-2177, 1979.

30. Masood S: Neoadjuvant chemotherapy in breast cancers. Womens Health (Lond) 12: 480-491, 2016.

31. Borkowska A, Knap N and Antosiewicz J: Diallyl trisulfide is more cytotoxic to prostate cancer cells PC-3 than to noncancerous epithelial cell line PNT1A: A possible role of p66Shc signaling axis. Nutr Cancer 65: 711-717, 2013.

32. Pan Y, Lin S, Xing R, Zhu M, Lin B, Cui J, Li W, Gao J, Shen L, Zhao Y, et al: Epigenetic upregulation of metallothionein $2 \mathrm{~A}$ by diallyl trisulfide enhances chemosensitivity of human gastric cancer cells to docetaxel through attenuating NF- $\kappa \mathrm{B}$ activation. Antioxid Redox Signal 24: 839-854, 2016.

33. Li W, Tian H, Li L, Li S, Yue W, Chen Z, Qi L, Hu W, Zhu Y, Hao B, et al: Diallyl trisulfide induces apoptosis and inhibits proliferation of A549 cells in vitro and in vivo. Acta Biochim Biophys Sin (Shanghai) 44: 577-583, 2012.
34. Kim SH, Kaschula CH, Priedigkeit N, Lee AV and Singh SV: Forkhead box Q1 is a novel target of breast cancer stem cell inhibition by diallyl trisulfide. J Biol Chem 291: 13495-13508, 2016.

35. Lai KC, Hsu SC, Yang JS, Yu CC, Lein JC and Chung JG: Diallyl trisulfide inhibits migration, invasion and angiogenesis of human colon cancer HT-29 cells and umbilical vein endothelial cells, and suppresses murine xenograft tumour growth. J Cell Mol Med 19: 474-484, 2015.

36. Oommen S, Anto RJ, Srinivas G and Karunagaran D: Allicin (from garlic) induces caspase-mediated apoptosis in cancer cells. Eur J Pharmacol 485: 97-103, 2004.

37. Xu L, Yu J, Zhai D, Zhang D, Shen W, Bai L, Cai Z and Yu C: Role of JNK activation and mitochondrial bax translocation in allicin-induced apoptosis in human ovarian cancer SKOV3 cells. Evid Based Complement Alternat Med 2014: 378684, 2014.

38. Li C, Lun W, Zhao X, Lei S, Guo Y, Ma J and Zhi F: Allicin alleviates inflammation of trinitrobenzenesulfonic acid-induced rats and suppresses P38 and JNK pathways in Caco-2 cells. Mediators Inflamm 2015: 434692, 2015.

39. Tufi R, Panaretakis T, Bianchi K, Criollo A, Fazi B, Di Sano F, Tesniere A, Kepp O, Paterlini-Brechot P, Zitvogel L, et al: Reduction of endoplasmic reticulum $\mathrm{Ca}^{2+}$ levels favors plasma membrane surface exposure of calreticulin. Cell Death Differ 15: 274-282, 2008.

40. Chao MP, Jaiswal S, Weissman-Tsukamoto R, Alizadeh AA, Gentles AJ, Volkmer J, Weiskopf K, Willingham SB, Raveh T, Park CY, et al: Calreticulin is the dominant pro-phagocytic signal on multiple human cancers and is counterbalanced by CD47. Sci Transl Med 2: 63-94, 2010.

41. No authors listed: Calreticulin inhibits commitment to adipocyte differentiation. J Cell Biol 208: 249-250, 2015.

42. Martín N, Bardisa L, Pantoja C, Barra E, Demetrio C, Valenzuela J, Barrios M and Sepúlveda MJ: Involvement of calcium in the cardiac depressant actions of a garlic dialysate. J Ethnopharmacol 55: 113-118, 1997. International (CC BY-NC-ND 4.0) License. 